\title{
Close-to-equilibrium behaviour of quadratic reaction-diffusion systems with detailed balance
}

\author{
María J. Cáceres José A. Cañizo
}

February 2017

\begin{abstract}
We study general quadratic reaction-diffusion systems with detailed balance, in space dimension $d \leq 4$. We show that close-to-equilibrium solutions (in an $L^{2}$ sense) are regular for all times, and that they relax to equilibrium exponentially in a strong sense. That is: all detailed balance equilibria are exponentially asymptotically stable in all $L^{p}$ norms, at least in dimension $d \leq 4$. The results are given in detail for the four-species reaction-diffusion system, where the involved constants can be estimated explicitly. The main novelty is the regularity result and exponential relaxation in $L^{p}$ norms for $p>1$, which up to our knowledge is new in dimensions 3 and 4 .
\end{abstract}

\section{Contents}

1 Introduction 1

2 Preliminaries and main results 4

3 Linearised system and spectral gap 10

4 Local $L^{2}$ stability $\quad 13$

$5 \quad$ Regularity of close-to-equilibrium solutions $\quad 15$

6 General quadratic systems with detailed balance 18

6.1 General setting and main result . . . . . . . . . . . . . . . . . . 19

6.2 The linearised system . . . . . . . . . . . . . . . . . 22

6.3 Local $L^{p}$ stability . . . . . . . . . . . . . . . . . . . . . . . 24

\section{Introduction}

Systems of reaction-diffusion equations model a wide variety of phenomena, and prominent among them is the behaviour of reacting chemical mixtures. The mathematical theory of these systems is far from complete, and in particular the existence of global 
regular solutions is unknown in many important cases. If we consider $I \geq 2$ species, denoted $A_{1}, \ldots, A_{I}$, undergoing a number $R \geq 1$ of different reactions

$$
\alpha_{1}^{r} A_{1}+\cdots+\alpha_{N}^{r} A_{I} \underset{k_{b}^{r}}{\stackrel{k_{f}^{r}}{\rightleftharpoons}} \beta_{1}^{r} A_{1}+\cdots+\beta_{N}^{r} A_{I}, \quad r=1, \ldots, R,
$$

then the equation satisfied by the concentrations $a_{i}=a_{i}(t, x)$ of the $A_{i}$ is

$$
\begin{aligned}
& \partial_{t} a_{i}=d_{i} \Delta a_{i}-R_{i}(a), \quad i=1, \ldots, I, \\
& \nabla_{x} a_{i}(t, x) \cdot \nu(x)=0, \quad t>0, x \in \partial \Omega, i=1, \ldots, I .
\end{aligned}
$$

The positive numbers $k_{f}^{r}$ and $k_{b}^{r}$, for $r=1 \ldots, R$, denote the forward and backward reaction rates, respectively, for each of the $R$ reactions. The vectors $\alpha^{r}=\left(\alpha_{1}^{r}, \ldots, \alpha_{I}^{r}\right)$ and $\beta^{r}=\left(\beta_{1}^{r}, \ldots, \beta_{I}^{r}\right)$ are the stoichiometric coefficients which specify the number of particles of each species that take part in each reaction, and the reaction term $R_{i}(a)$ depends on $a=\left(a_{i}\right)_{i=1, \ldots, I}$ and is obtained from the law of mass action. Complete details on this setting are given in Section 6, and for the moment we omit them for brevity. A very interesting basic model that presents the main difficulties is the following, sometimes called the four species model: consider a set of four chemical substances $A_{1}, A_{2}, A_{3}, A_{4}$ which undergo the reactions

$$
A_{1}+A_{3} \underset{k_{2}}{\stackrel{k_{1}}{\rightleftharpoons}} A_{2}+A_{4}
$$

at positive rates $k_{1}, k_{2}$ as marked. We assume these substances are confined to a domain $\Omega \subseteq \mathbb{R}^{d}$ (a connected, bounded, open region with smooth boundary — at least $\mathcal{C}^{2+\alpha}$ with $\alpha>0)$, and we denote the concentration of $A_{i}$ by $a_{i}=a_{i}(t, x)$, depending on time $t \geq 0$ and space $x \in \Omega$. Except in Section 6, it is understood that the index $i$ always ranges from 1 to 4 . If these substances also diffuse in a domain with diffusion constant $d_{i}$ corresponding to $A_{i}$ then the following system models the time evolution of the concentrations $a_{i}$ :

$$
\left.\begin{array}{l}
\partial_{t} a_{1}=d_{1} \Delta a_{1}-k_{1} a_{1} a_{3}+k_{2} a_{2} a_{4}, \\
\partial_{t} a_{2}=d_{2} \Delta a_{2}+k_{1} a_{1} a_{3}-k_{2} a_{2} a_{4}, \\
\partial_{t} a_{3}=d_{3} \Delta a_{3}-k_{1} a_{1} a_{3}+k_{2} a_{2} a_{4}, \\
\partial_{t} a_{4}=d_{4} \Delta a_{4}+k_{1} a_{1} a_{3}-k_{2} a_{2} a_{4},
\end{array}\right\} \quad t>0, x \in \Omega .
$$

We always assume that all $d_{i}$ are strictly positive. We also assume no-flux boundary conditions which ensure that the total mass is conserved:

$$
\nabla_{x} a_{i}(t, x) \cdot \nu(x)=0, \quad t>0, x \in \partial \Omega, i=1, \ldots, 4,
$$

where $\nu(x)$ denotes the outer normal to the boundary of $\Omega$ at point $x$. The system (4) is quadratic in the nonlinearities and satisfies the detailed balance condition: there is a space-homogeneous equilibrium $\left(a_{i, \infty}\right)_{i=1, \ldots, 4}$ which makes each of the reactions balanced, that is, it satisfies

$$
k_{1} a_{1, \infty} a_{3, \infty}=k_{2} a_{2, \infty} a_{4, \infty} .
$$

Since in this case there is only one reaction, it is obvious that all space-homogeneous equilibria must satisfy this. In general, when detailed balance holds one can show that all equilibria must be space-homogeneous and satisfy the same condition. 
The existence of solutions and asymptotic behaviour of the system (4)-(5) and in general (1)-(2) have been studied in a number of works, and several previous results in reaction-diffusion systems apply to it. In general, difficulties increase with the strength of the nonlinearities and the space dimension. It is known that weak solutions to (4)(5) in $L^{2}([0, T) \times \Omega)$ exist in all dimensions and for all $T>0$, and in general weak $L^{2}$ solutions to (1)-(2) exist as long as the system is at most quadratic and satisfies the detailed balance condition (Desvillettes et al., 2007). In this paper we always work with this concept of solution. A general theory of renormalised solutions for entropydissipating systems that does not have the restriction of the system being quadratic has recently been developed in Fischer (2015). Classical solutions are more elusive: they exist for a short time thanks to general theory of parabolic equations (Amann, 1985), and global-in-time classical solutions are relatively well understood in a few cases. For the system (4)-(5) the current situation is the following:

1. Global regular solutions are known to exist in space dimension $d \leq 2$ (Cañizo, Desvillettes, and Fellner, 2014; Desvillettes and Fellner, 2006, 2008; Goudon and Vasseur, 2010).

2. Global regular solutions also exist in any space dimension whenever the nonlinearities are of degree $<2$ (Caputo and Vasseur, 2009). Notice that this does not apply to system (4), which has quadratic nonlinearities.

3. Regular solutions are also understood in any space dimension when diffusion coefficients are not too far from each other (Cañizo, Desvillettes, and Fellner, 2014; Fellner and Laamri, 2016; Fellner, Latos, and Suzuki, 2016). They are also understood if the diffusion coefficients satisfy $d_{1}=d_{2}$ and $d_{3}=d_{4}$ (Fougères et al., 2014; Gentil and Zegarlinski, 2010).

4. Finally, there are some special cases where the nonlinearities contain some linear terms and allow us to study the regularity of some of the $a_{i}$ separately, and then bootstrap the regularity of the other ones (see Desvillettes and Fellner (2006), and Elias (2016) for a recent application of this idea).

In general it is not known whether global classical solutions exist for all smooth initial conditions. Regarding asymptotic behaviour, it is expected that solutions converge exponentially fast to equilibrium when detailed balance holds. Entropy methods have been successfully applied to this kind of systems in almost complete generality, obtaining exponential convergence to equilibrium in the entropic sense and in $L^{1}$ (Desvillettes and Fellner, 2006, 2008; Fellner and Tang, 2017; Gentil and Zegarlinski, 2010). In some cases one may also obtain $L^{2}$ convergence by using specifically designed functionals (see Rionero $(2006,2015)$ and the references therein). Relaxation to equilibrium is also understood for linear systems without detailed balance (Fellner et al., 2015). Once a rate of relaxation to equilibrium in entropy has been obtained, any slowly-growing $a$ priori bounds on the solution in $L^{p}$ spaces or on its regularity yield convergence to equilibrium in a stronger sense (see Desvillettes and Fellner (2008) and the technique we use to prove Theorem 2.1).

In this paper we study a natural regime which seems to be mostly missing in the literature: that of close-to-equilibrium solutions. Some attention has been devoted to this (for example (Smoller, 1994)) but a general result for detailed balance systems 
is, to our knowledge, lacking. Our main result is that close-to-equilibrium solutions (i.e., whose initial condition is $L^{2}$-close to the equilibrium) in dimension $d \leq 4$ are regular and converge to equilibrium exponentially fast in all $L^{p}$ norms including $L^{\infty}$, which can readily be extended to exponential convergence in higher Sobolev norms. Notice that closeness to equilibrium is the main assumption, and no requirements on the diffusion coefficients $d_{i}$ are made except for their positivity. The regularity result is the main novelty, since exponential convergence to equilibrium can be obtained from it by using simple interpolation techniques (Desvillettes and Fellner, 2006; Fellner and Tang, 2017). However, in the close-to-equilibrium regime we show that this can be obtained by simpler methods than in the general case. We do not know whether the limitation that $d \leq 4$ is essential or not.

The natural driving idea is that in the close-to-equilibrium regime the behaviour is dominated by the linearisation of (4) near an equilibrium. We give fully detailed arguments in the case of the four-species model in Sections 2-5, and later in Section 6 we extend these ideas to general quadratic equations with detailed balance. According to this, the paper is structured as follows: in Section 2 we gather our precise assumptions, notation and some preliminary results that are needed later. In Section 3 we briefly study the linearised system around an equilibrium, mainly gathering results which are already known in the literature. Section 4 contains estimates on the exponential relaxation of the $L^{2}$ distance to equilibrium, and these estimates are finally used in Section 5 in order to obtain a priori estimates of higher $L^{p}$ norms, and strong convergence to equilibrium. The $L^{\infty}$ estimates in particular show that close-to-equilibrium solutions are regular. In Section 6 we state and prove our theorem on general quadratic reaction-diffusion systems with detailed balance.

\section{Preliminaries and main results}

Notation. Most of the functions we use depend on time $t \geq 0$ and space $x \in \Omega$. When integrating we often omit the variables $(t, x)$, and they are assumed unless we explicitly say otherwise. The differential operators $\nabla$ and $\Delta$ always act on the variable $x$. When $\Omega$ is regular enough, $\nu(x)$ denotes the exterior normal to $\Omega$ at a point $x \in \partial \Omega$. As usual, we denote by $L^{p}(U)$ the Lebesgue space of real $p$-integrable functions on a Borel measurable set $U \subseteq \mathbb{R}^{m}$, for $1 \leq p \leq \infty$, and the associated norm by $\|\cdot\|_{p}$. We consider this norm defined for every measurable function, with its value possibly being equal to $+\infty$. We will often deal with vectors $h=\left(h_{1}, h_{2}, h_{3}, h_{4}\right) \in\left(L^{p}(\Omega)\right)^{4}$, with $\Omega \subseteq \mathbb{R}^{d}$ an open set, and then we usually denote

$$
\|h\|_{p}^{p}:=\sum_{i=1}^{4} \int_{\Omega} \frac{\left|h_{i}\right|^{p}}{a_{i, \infty}^{p-1}} \mathrm{~d} x, \quad\|\nabla h\|_{p}^{p}:=\sum_{j=1}^{d} \sum_{i=1}^{4} \int_{\Omega} \frac{\left|\partial_{j} h_{i}\right|^{p}}{a_{i, \infty}^{p-1}} \mathrm{~d} x,
$$

where $\left(a_{i, \infty}\right)_{i=1, \ldots, 4}$ are numbers defined below, and represent the equilibrium values of the system (4)-(5). In the case of $p=2$, the associated scalar product is

$$
\langle h, f\rangle:=\sum_{i=1}^{4} \int_{\Omega} \frac{h_{i} f_{i}}{a_{i, \infty}} \mathrm{d} x \quad \text { for } h, f \in\left(L^{2}(\Omega)\right)^{4},
$$

for any $h, f \in\left(L^{2}(\Omega)\right)^{4}$. This weighted definition of $\|h\|_{p}$ is especially natural for the $L^{2}$ norm, which is a Lyapunov functional for (4)-(5). Any other weights could be used 
for $p \neq 2$, but we keep these for consistency. It should not cause any confusion with the usual notation for $L^{p}$ norms, since (6) is only used for vectors in $\left(L^{p}(\Omega)\right)^{4}$.

If $I \subseteq \mathbb{R}$ is an interval, we denote by $L^{p}\left(I, L^{q}(\Omega)\right)$ the set of measurable functions $u: I \rightarrow L^{q}(\Omega)$ such that $\|u\|_{q}$ is in $L^{p}(I)$.

Main assumptions. Since the reaction constants $k_{1}, k_{2}$ are not essential to our results, let us set $k_{1}=k_{2}=1$ throughout to simplify the notation. Similarly, we assume that $|\Omega|=1$ (where $|\Omega|$ denotes its Lebesgue measure), in addition to the regularity hypotheses mentioned in the introduction. All of our results can easily be extended to the case of general positive constants $k_{1}, k_{2}$ and a general bounded domain $\Omega$ of any size.

Hypothesis 2.1. The region $\Omega \subseteq \mathbb{R}^{d}$ is nonempty, open, connected, bounded and with boundary of class $\mathcal{C}^{2+\alpha}$ for some $\alpha>0$. We also assume $|\Omega|=1$.

This ensures that the heat equation is well posed in the domain $\Omega$ with Neumann boundary conditions and avoids non-essential technicalities. We also need to assume that the diffusion constants are strictly positive:

Hypothesis 2.2. We assume $d_{1}, d_{2}, d_{3}, d_{4}$ are strictly positive real numbers.

Also, and we always consider nonnegative initial conditions which ensure that the equilibrium is strictly positive (see below):

Hypothesis 2.3. The functions $a_{1,0}, a_{2,0}, a_{3,0}, a_{4,0}: \Omega \rightarrow \mathbb{R}$ are in $L^{2}(\Omega)$, are nonnegative, and satisfy

$$
\left(\int_{\Omega} a_{1,0} \mathrm{~d} x\right)\left(\int_{\Omega} a_{3,0} \mathrm{~d} x\right)+\left(\int_{\Omega} a_{2,0} \mathrm{~d} x\right)\left(\int_{\Omega} a_{4,0} \mathrm{~d} x\right)>0
$$

Equivalently: either $a_{1,0}$ and $a_{3.0}$ are both nonzero, or $a_{2,0}$ and $a_{4,0}$ are both nonzero. This ensures that there are enough reactants for at least one of the reactions in (3) to happen, and ensures that the equilibrium is positive.

With our simplification that $k_{1}=k_{2}=1$, equation (4) becomes

$$
\left.\begin{array}{l}
\partial_{t} a_{1}=d_{1} \Delta a_{1}-a_{1} a_{3}+a_{2} a_{4}, \\
\partial_{t} a_{2}=d_{2} \Delta a_{2}+a_{1} a_{3}-a_{2} a_{4}, \\
\partial_{t} a_{3}=d_{3} \Delta a_{3}-a_{1} a_{3}+a_{2} a_{4}, \\
\partial_{t} a_{4}=d_{4} \Delta a_{4}+a_{1} a_{3}-a_{2} a_{4},
\end{array}\right\} \quad t>0, x \in \Omega,
$$

always with Neumann boundary conditions

$$
\nabla_{x} a_{i}(t, x) \cdot \nu(x)=0, \quad t>0, x \in \partial \Omega, i=1, \ldots, 4,
$$

and with initial conditions given by the $a_{i, 0}$ :

$$
a_{i}(0, x)=a_{i, 0}(x) \quad x \in \Omega, i=1, \ldots, 4 .
$$

We sometimes denote $a=\left(a_{1}, a_{2}, a_{3}, a_{4}\right)$, we write $D(a)=\left(d_{i} \Delta a_{i}\right)_{i=1, \ldots, 4}$ and denote by $N(a)=\left(N_{1}(a), N_{2}(a), N_{3}(a), N_{4}(a)\right)$ the nonlinear terms on the right hand side of $(8)$, so that $(8)$ can be written as

$$
\partial_{t} a=D(a)+N(a)
$$


Concept of solution. Take $T \in(0,+\infty]$. As is standard we define a mild solution (also referred to as weak solution by some authors) of the system (8)-(10) on $[0, T)$ to be a set of four measurable functions $a_{i}:[0, T) \times \Omega \rightarrow \mathbb{R}, i=1, \ldots, 4$, such that the products $a_{1} a_{3}$ and $a_{2} a_{4}$ are in $L^{1}([0, t) \times \Omega)$ for all $0<t<T$, and which satisfy

$$
a_{i}(t, x)=e^{t d_{i} \Delta} a_{i, 0}(t, x)+\int_{0}^{t} e^{(t-s) d_{i} \Delta} N_{i}(a(s, x)) \mathrm{d} s, \quad i=1, \ldots, 4
$$

almost everywhere on $[0, T) \times \Omega$, where $e^{t \Delta}$ denotes the semigroup associated to the heat equation on $\Omega$ with Neumann boundary conditions. A classical solution or regular solution is a set of four functions $a_{i} \in \mathcal{C}([0, T) \times \bar{\Omega}), i=1, \ldots, 4$ such that

$$
\Delta a_{i}, \partial_{t} a_{i} \in \mathcal{C}^{2}((0, T) \times \bar{\Omega}), \quad \mathrm{i}=1, \ldots, 4,
$$

and which satisfy (8)-(10) pointwise.

Equilibria and conserved quantities. The system (8)-(9) has a three-dimensional space of conserved quantities which is generated for example by

$$
M_{12}:=\int_{\Omega}\left(a_{1}+a_{2}\right) \mathrm{d} x, \quad M_{14}:=\int_{\Omega}\left(a_{1}+a_{4}\right) \mathrm{d} x \quad \text { and } \quad M_{32}:=\int_{\Omega}\left(a_{3}+a_{2}\right) \mathrm{d} x .
$$

Other conserved quantities can be obtained from these, such as for example the total mass

$$
M:=\int_{\Omega}\left(a_{1}+a_{2}+a_{3}+a_{4}\right) \mathrm{d} x .
$$

All of these quantities are formally constant (their time derivative is 0 ). Given nonnegative initial conditions $a_{i, 0} \in L^{1}(\Omega), i=1, \ldots, 4$, there exists a unique positive equilibrium which we denote by $a_{i, \infty}, i=1, \ldots, 4$, having the same invariants as the solution; that is, there is a unique set of four positive numbers $\left(a_{i, \infty}\right)_{i=1, \ldots, 4}$ such that

$$
\begin{gathered}
a_{1, \infty} a_{3, \infty}=a_{2, \infty} a_{4, \infty}, \\
a_{1, \infty}+a_{2, \infty}=\int_{\Omega}\left(a_{1,0}+a_{2,0}\right) \mathrm{d} x=M_{12}, \\
a_{1, \infty}+a_{4, \infty}=\int_{\Omega}\left(a_{1,0}+a_{4,0}\right) \mathrm{d} x=M_{14}, \\
a_{2, \infty}+a_{3, \infty}=\int_{\Omega}\left(a_{2,0}+a_{3,0}\right) \mathrm{d} x=M_{32} .
\end{gathered}
$$

Explicitly,

$$
\begin{array}{ll}
a_{1, \infty}=\frac{M_{12} M_{14}}{M}, & a_{2, \infty}=\frac{M_{12} M_{32}}{M}, \\
a_{3, \infty}=\frac{M_{32} M_{34}}{M}, & a_{4, \infty}=\frac{M_{14} M_{34}}{M} .
\end{array}
$$

Observe that Hypothesis 2.3 ensures that $M \neq 0$, and that the $a_{i, \infty}$ are strictly positive, since it ensures that all of $M_{12}, M_{14}, M_{32}$ and $M_{34}$ are positive. 
Main result. Our main result for the four-species system can be summarised as follows:

Theorem 2.1. Assume Hypotheses 2.1, 2.2 and 2.3, and let $d \leq 4$. Let $\left(a_{i}\right)_{i=1, \ldots, 4}$ be a solution to the system (8)-(9). Let $a_{i, \infty}, i=1, \ldots, 4$, denote the only positive equilibrium of (4) with the same invariants as $\left(a_{i, 0}\right)_{i=1, \ldots, 4}$ (that is, satisfying (12)).

Then for any $2 \leq p<\infty$, assuming $\left\|a_{0}\right\|_{p}<+\infty$, there exist positive constants $\lambda, K, \epsilon>0$ depending on $p, d,\left(d_{i}\right)_{i=1, \ldots, 4},\left(a_{i, \infty}\right)_{i=1, \ldots, 4}, \Omega$ and $\left\|a_{0}\right\|_{p}$ such that

$$
\sum_{i=1}^{4} \int_{\Omega} \frac{\left|a_{i}(t, x)-a_{i, \infty}\right|^{p}}{a_{i, \infty}} \mathrm{d} x \leq K e^{-\lambda t}
$$

whenever the $a_{i, 0}$ satisfy

$$
\sum_{i=1}^{4} \int_{\Omega} \frac{\left|a_{i, 0}(x)-a_{i, \infty}\right|^{2}}{a_{i, \infty}} \mathrm{d} x \leq \epsilon .
$$

As a consequence, if $a_{i, 0} \in L^{\infty}(\Omega)$, then the solution to (8)-(9) is uniformly bounded in $L^{\infty}(\Omega)$, and is a classical solution for $t>0$.

Its proof is given at the end of Section 5. We notice that Theorem 2.1 contains both a regularity result and exponential decay of all $L^{p}$ norms (for $p<\infty$ ). As discussed in the introduction, its main novelty is the regularity of solutions and decay in $L^{p}$ norms for $p>1$, since decay in $L^{p}$ for $p=1$ was already known in previous works (Desvillettes and Fellner, 2006, 2008).

This result can be extended with almost the same proof to a general quadratic reaction-diffusion system with detailed balance of the form (1)-(2), always in dimension $d \leq 4$. Full details are given in Section 6 and here we just highlight our main result in this general framework. For the precise assumptions of the above result we refer to Section 6, but their essence is that the system needs to satisfy detailed balance, be at most quadratic, and otherwise satisfy conditions analogous to Hypotheses 2.1-2.3:

Theorem 2.2. Assume Hypothesis 2.1 and Hypotheses 6.1-6.4 (cf. Section 6), and let $d \leq 4$. Let $\left(a_{i}\right)_{i=1, \ldots, I}$ be a solution to the system (1)-(2) with initial condition $a_{0}=\left(a_{0, i}\right)_{i=1, \ldots, I}$. Let $a_{i, \infty}, i=1, \ldots, I$, denote a detailed balance equilibrium of $(1)$ with the same invariants as $\left(a_{i, 0}\right)_{i=1, \ldots, I}$.

Then for any $2 \leq p<\infty$ there exist positive constants $\lambda, K, \epsilon>0$ depending on $p$, $d,\left(d_{i}\right)_{i=1, \ldots, I},\left(a_{i, \infty}\right)_{i=1, \ldots, I}, \Omega$ and $\left\|a_{0}\right\|_{p}$ such that

$$
\sum_{i=1}^{I} \int_{\Omega} \frac{\left|a_{i}(t, x)-a_{i, \infty}\right|^{p}}{a_{i, \infty}} \mathrm{d} x \leq K e^{-\lambda t}
$$

whenever the $a_{i, 0}$ satisfy

$$
\sum_{i=1}^{I} \int_{\Omega} \frac{\left|a_{i, 0}(x)-a_{i, \infty}\right|^{2}}{a_{i, \infty}} \mathrm{d} x \leq \epsilon .
$$

As a consequence, if $a_{i, 0} \in L^{\infty}(\Omega)$, then the solution to (47)-(49) is uniformly bounded in $L^{\infty}(\Omega)$, and is a classical solution for $t>0$. 
The main restrictions and shortcomings of this result are the following:

1. As opposed to Theorem 2.1, the constant $\lambda$ cannot be estimated constructively in Theorem 2.2. Both results rely on a spectral gap of the linearised system. In full generality one can prove the existence of this positive spectral gap, but we can give no estimate on its size. In this generality, estimating this spectral gap in any constructive way is a research area by itself and is out of the scope of this paper.

2. The proof only works in dimension $d \leq 4$, and for reactions which involve at most two reactants on each side of the reaction. The reason for this, as in the four-species case, is that the argument showing the nonlinear perturbations close to equilibrium are negligible relies on Sobolev embedding estimates which lead to a restriction on the exponents.

3. The proof works only for the stability of a detailed balance equilibrium, for which the relative entropy (or relative free energy) is a known Lyapunov functional of the system. It is possible that these results can be extended to systems with complex balance, for which there is still an entropy structure, but we have not followed that extension.

On the other hand, with the above restrictions the result is fully general: detailed balance equilibria for quadratic systems in dimension $d \leq 4$ are always exponentially asymptotically stable, at least in a neighbourhood of the equilibrium. This applies even if more than one equilibrium exists, in particular when boundary equilibria exist (equilibria with some component equal to 0). This local stability is known to hold for the system without diffusion (Horn and Jackson, 1972), so our contribution is the observation that diffusion does not change this behaviour for quadratic systems, in dimension $d \leq 4$.

Entropy. Since the system (8) (or (4)) satisfies the detailed balance condition, it is well-known that the relative free energy is nonincreasing: if we define it by

$$
H\left(a \mid a_{\infty}\right):=\sum_{i=1}^{4} \int_{\Omega}\left(a_{i} \log \frac{a_{i}}{a_{i, \infty}}-a_{i}+a_{i, \infty}\right)
$$

then we have

$$
\frac{\mathrm{d}}{\mathrm{d} t} H\left(a \mid a_{\infty}\right)=-\sum_{i=1}^{4} \int_{\Omega} d_{i} \frac{\left|\nabla a_{i}\right|^{2}}{a_{i}} \mathrm{~d} x-\int_{\Omega} \Psi\left(a_{1} a_{3}, a_{2} a_{4}\right) \mathrm{d} x
$$

for any solution $a$ to (8)- (9), where

$$
\Psi(a, b):=(a-b)(\log a-\log b)
$$

for $a, b>0$.

Gagliardo-Nirenberg inequalities. A basic tool is the so-called Gagliardo-Nirenberg inequality, which we state on a bounded domain $\Omega$, and in the particular case we will use. This statement can be found in Nirenberg (1959, pp. 125-126); see also Friedman (2008, Theorem 9.3): 
Lemma 2.3 (Gagliardo-Nirenberg inequality). Let $\Omega \subseteq \mathbb{R}^{d}$ be a nonempty, open, bounded domain with Lipschitz boundary. Take $1 \leq q, s<\infty$ and assume $p, \theta$ satisfy

$$
\frac{1}{p}=\theta\left(\frac{1}{q}-\frac{1}{d}\right)+(1-\theta) \frac{1}{s}, \quad 0 \leq \theta<1, \quad 1 \leq p<+\infty .
$$

Then there exists a constant $C>0$ depending only on $d, q, r, p$ and the domain $\Omega$ such that

$$
\|u\|_{p} \leq C\|\nabla u\|_{q}^{\theta}\|u\|_{s}^{(1-\theta)}+C\|u\|_{1}
$$

for all $u \in L^{1}(\Omega)$.

Remark 2.4. Observe that the right hand side, or both sides, may be equal to $+\infty$. In the above theorem we have avoided the special cases $\theta=1$ or $q=\infty$, which require additional hypotheses to hold and will not be used in this paper. The term $\|u\|_{1}$ on the right hand side is needed due to the fact that we are on a bounded domain (otherwise a nonzero constant is a counterexample to the inequality). Other versions of this inequality in a bounded domain involve the Sobolev norm in $W^{1, q}$ instead of $\|\nabla u\|_{q}$ (see Brezis (2010, p. 233)), and it is likely that the same statement holds without the $\|u\|_{1}$ term if one writes $u-\bar{u}$ instead of $u$ on the left hand side, with $\bar{u}:=\frac{1}{|\Omega|} \int_{\Omega} u$. We have not been able to find a precise reference that contains these results in full generality.

Parabolic regularisation of the heat equation. We will use some relatively wellknown results on regularisation in parabolic equations. For completeness, and since precise references for some of them are not easily found, we gather them here. We first state a standard $L^{p}-L^{q}$ regularisation property of the heat equation, and then use it to show $L^{\infty}$ regularisation of the heat equation with a source term under certain conditions.

Lemma 2.5. Assume $\Omega \subseteq \mathbb{R}^{d}$ satisfies Hypothesis 2.1. Consider the solution $u$ to the heat equation on $\Omega$ with Neumann boundary conditions and initial condition $u_{0} \in L^{q}(\Omega)$, with $1 \leq q<\infty$ :

$$
\left.\begin{array}{ll}
\partial_{t} u=\Delta u, & t>0, x \in \Omega, \\
\nabla u \cdot \nu=0, & t>0, x \in \partial \Omega \\
u(0, x)=u_{0}(x), & x \in \Omega .
\end{array}\right\}
$$

For every $r$ with $q \leq r \leq \infty$ it holds that

$$
\|u(t, \cdot)\|_{r} \leq C\left\|u_{0}\right\|_{q}\left(1+t^{-\frac{d}{2}\left(\frac{1}{q}-\frac{1}{r}\right)}\right) \quad \text { for all } t>0,
$$

for some constant $C=C(d, r, q)>0$.

Lemma 2.6. Assume $\Omega \subseteq \mathbb{R}^{d}$ satisfies Hypothesis 2.1, and take $f \in L_{\mathrm{loc}}^{\infty}\left([0, \infty), L^{q}(\Omega)\right)$ for some $1 \leq q \leq \infty$. Consider the solution $u$ to the following heat equation on $\Omega$ with a source term $f$ and initial condition $u_{0} \in L^{\infty}(\Omega)$ :

$$
\left.\begin{array}{ll}
\partial_{t} u=\Delta u+f, & t>0, x \in \Omega, \\
\nabla u \cdot \nu=0, & t>0, x \in \partial \Omega \\
u(0, x)=u_{0}(x), & x \in \Omega .
\end{array}\right\}
$$

If $q>d / 2$ then $u \in L_{\mathrm{loc}}^{\infty}\left([0,+\infty), L^{\infty}(\Omega)\right)$. 
Proof. We use Duhamel's formula to write

$$
u(t, x)=\left(e^{t \Delta} u_{0}\right)(x)+\int_{0}^{t}\left(e^{(t-s) \Delta} f_{s}\right)(x) \mathrm{d} s,
$$

where $f_{s}(x) \equiv f(s, x)$ and $e^{t \Delta}$ denotes the heat semigroup on $L^{1}(\Omega)$ at time $t \geq 0$ with Neumann boundary conditions. Taking the $L^{\infty}$ norm and using both Lemma 2.5 and the maximum principle for the heat semigroup,

$$
\begin{aligned}
\|u(t, \cdot)\|_{\infty} \leq\left\|u_{0}\right\|_{\infty} & +\int_{0}^{t}\left\|e^{(t-s) \Delta} f_{s}\right\|_{\infty} \mathrm{d} s \\
& \leq\left\|u_{0}\right\|_{\infty}+C \int_{0}^{t}\left(1+(t-s)^{-\frac{d}{2 q}}\right)\left\|f_{s}\right\|_{q} \mathrm{~d} s \\
& \leq\left\|u_{0}\right\|_{\infty}+C C_{f} \int_{0}^{t}\left(1+(t-s)^{-\frac{d}{2 q}}\right) \mathrm{d} s,
\end{aligned}
$$

where $C_{f}$ is the norm of $f$ in $L^{\infty}\left((0, \infty), L^{q}\left(\mathbb{R}^{d}\right)\right)$. The assumption that $q>d / 2$ shows that the last integral is finite and gives the $L^{\infty}$ bound on the solution.

\section{$3 \quad$ Linearised system and spectral gap}

We begin our study by considering the linearisation of (8) around the equilibrium $\left(a_{i, \infty}\right)_{i=1, \ldots, 4}$. If we set

$$
a_{i}=a_{i, \infty}+h_{i}, \quad i=1, \ldots, 4,
$$

then the perturbations from equilibrium $h_{i}$ satisfy the following system of equations to first order:

$$
\left.\begin{array}{l}
\partial_{t} h_{1}=d_{1} \Delta h_{1}-a_{3, \infty} h_{1}-a_{1, \infty} h_{3}+a_{4, \infty} h_{2}+a_{2, \infty} h_{4}, \\
\partial_{t} h_{2}=d_{2} \Delta h_{2}+a_{3, \infty} h_{1}+a_{1, \infty} h_{3}-a_{4, \infty} h_{2}-a_{2, \infty} h_{4}, \\
\partial_{t} h_{3}=d_{3} \Delta h_{3}-a_{3, \infty} h_{1}-a_{1, \infty} h_{3}+a_{4, \infty} h_{2}+a_{2, \infty} h_{4}, \\
\partial_{t} h_{4}=d_{4} \Delta h_{4}+a_{3, \infty} h_{1}+a_{1, \infty} h_{3}-a_{4, \infty} h_{2}-a_{2, \infty} h_{4},
\end{array}\right\}
$$

for $t>0, x \in \Omega$, with the same boundary conditions:

$$
\nabla_{x} h_{i}(t, x) \cdot \nu(x)=0, \quad t>0, x \in \partial \Omega, i=1, \ldots, 4 .
$$

The conserved quantities of system (8) translate into the following property for $\left(h_{i}\right)_{i}$ :

$$
\int_{\Omega}\left(h_{1}+h_{2}\right) \mathrm{d} x=\int_{\Omega}\left(h_{1}+h_{4}\right) \mathrm{d} x=\int_{\Omega}\left(h_{2}+h_{3}\right) \mathrm{d} x=0 .
$$

In particular, $\sum_{i=1}^{4} \int_{\Omega} h_{i} \mathrm{~d} x=0$. We shorten the notation of system (17) in a similar way as in (11) by writing

$$
\partial_{t} h_{i}=d_{i} \Delta h_{i}+L_{i} h, \quad i=1, \ldots, 4,
$$

where $h \equiv\left(h_{1}, h_{2}, h_{3}, h_{4}\right)$ and

$$
L_{i} h:=(-1)^{i}\left(a_{3, \infty} h_{1}+a_{1, \infty} h_{3}-a_{4, \infty} h_{2}-a_{2, \infty} h_{4}\right) .
$$


Calling $L h:=\left(L_{1} h, L_{2} h, L_{3} h, L_{4} h\right)$ we may write this more abstractly as

$$
\partial_{t} h=D h+L h=: T h .
$$

Since the equations (4) satisfy the detailed balance conditions, the free energy of the system (8)-(9) is nonincreasing in time and the system (17)-(18) inherits a natural Lyapunov functional: one can check that (with the definition of $\|h\|_{2}$ given in (6))

$$
\begin{aligned}
\frac{\mathrm{d}}{\mathrm{d} t}\|h\|_{2}^{2} & =\frac{\mathrm{d}}{\mathrm{d} t} \sum_{i=1}^{4} \int_{\Omega} \frac{h_{i}^{2}}{a_{i, \infty}} \mathrm{d} x=2\langle h, T h\rangle \\
& =-2 \sum_{i=1}^{4} d_{i} \int_{\Omega} \frac{\left|\nabla h_{i}\right|^{2}}{a_{i, \infty}} \mathrm{d} x-2 C_{M} \int_{\Omega}\left(\frac{h_{1}}{a_{1, \infty}}+\frac{h_{3}}{a_{3, \infty}}-\frac{h_{2}}{a_{2, \infty}}-\frac{h_{4}}{a_{4, \infty}}\right)^{2} \mathrm{~d} x,
\end{aligned}
$$

where $C_{M}=\frac{M_{12} M_{32} M_{14} M_{34}}{M^{2}}$. We first observe that the linear operator $T$ satisfies the following inequality, sometimes known as a spectral gap inequality:

Lemma 3.1. Take numbers $a_{i, \infty}>0$ for $i=1, \ldots, 4$. Assume Hypotheses 2.1 and 2.2, and define the linear operator $T$ by (21)-(22). There exists $\lambda_{*}>0$ such that

$$
\langle h, T h\rangle \leq-\lambda_{*}\|h\|_{2}^{2}
$$

for all $h \in\left(L^{2}(\Omega)\right)^{4}$ satisfying (19). The constant $\lambda_{*}$ depends only on the dimension $d$, the domain $\Omega$, the numbers $a_{i, \infty}, i=1, \ldots, 4$ and the diffusion constants $d_{i}, i=1, \ldots, 4$.

The above result can be deduced from the entropy inequalities in Desvillettes and Fellner (2008), but we have not been able to find a precise reference that states it. We give a full proof in our particular case for completeness:

Proof of Lemma 3.1. If we denote

$$
\bar{h}_{i}:=\int_{\Omega} h_{i} \mathrm{~d} x
$$

then the conservation laws (19) allow us to write $\bar{h}_{i}$ in terms of $\bar{h}_{1}$ as

$$
\bar{h}_{i}=(-1)^{i+1} \bar{h}_{1} \text {. }
$$

In order to show the result we use the expression of $\langle h, T h\rangle$ given in (23). Due to the Poincaré inequality we have

$$
\begin{gathered}
\sum_{i=1}^{4} d_{i} \int_{\Omega} \frac{\left|\nabla h_{i}\right|^{2}}{a_{i, \infty}} \mathrm{d} x \geq C_{\Omega} \sum_{i=1}^{4} d_{i} \int_{\Omega} \frac{\left|h_{i}-\bar{h}_{i}\right|^{2}}{a_{i, \infty}} \mathrm{d} x \\
=C_{\Omega} \sum_{i=1}^{4} d_{i} \int_{\Omega} \frac{\left|h_{i}\right|^{2}}{a_{i, \infty}} \mathrm{d} x-C_{\Omega} \sum_{i=1}^{4} d_{i} \frac{\left|\bar{h}_{i}\right|^{2}}{a_{i, \infty}},
\end{gathered}
$$

where $C_{\Omega}$ is the Poincaré constant of the domain $\Omega$. For the second term in (23) it holds that

$$
\begin{aligned}
\int_{\Omega}\left(\frac{h_{1}}{a_{1, \infty}}+\frac{h_{3}}{a_{3, \infty}}-\frac{h_{2}}{a_{2, \infty}}-\frac{h_{4}}{a_{4, \infty}}\right)^{2} \mathrm{~d} x & \geq\left(\frac{\bar{h}_{1}}{a_{1, \infty}}+\frac{\bar{h}_{3}}{a_{3, \infty}}-\frac{\bar{h}_{2}}{a_{2, \infty}}-\frac{\bar{h}_{4}}{a_{4, \infty}}\right)^{2} \\
& =\left|\bar{h}_{1}\right|^{2}\left(\sum_{i=1}^{4} \frac{1}{a_{i, \infty}}\right)^{2} .
\end{aligned}
$$


Therefore, taking $\gamma$ small enough so that

$$
\gamma<\min \left\{C_{\Omega}, \frac{C_{M}\left(\sum_{i=1}^{4} \frac{1}{a_{i, \infty}}\right)^{2}}{\sum_{i=1}^{4} \frac{d_{i}}{a_{i, \infty}}}\right\}
$$

we have

$$
\begin{aligned}
-\langle h, T h\rangle & \geq \gamma\left(\sum_{i=1}^{4} d_{i} \int_{\Omega} \frac{\left|h_{i}\right|^{2}}{a_{i, \infty}} \mathrm{d} x-\left|\bar{h}_{1}\right|^{2} \sum_{i=1}^{4} \frac{d_{i}}{a_{i, \infty}}\right)+C_{M}\left|\bar{h}_{1}\right|^{2}\left(\sum_{i=1}^{4} \frac{1}{a_{i, \infty}}\right)^{2} \\
& \geq \gamma \min _{i \in\{1, \ldots, 4\}}\left\{d_{i}\right\}\|h\|_{2}^{2}+\left|\bar{h}_{1}\right|^{2}\left(C_{M}\left(\sum_{i=1}^{4} \frac{1}{a_{i, \infty}}\right)^{2}-\gamma \sum_{i=1}^{4} \frac{d_{i}}{a_{i, \infty}}\right) \\
& \geq \gamma \min _{i \in\{1, \ldots, 4\}}\left\{d_{i}\right\}\|h\|_{2}^{2},
\end{aligned}
$$

This shows the result.

Remark 3.2. Notice that this proof relies on the fact that all constants $d_{i}$ are strictly positive (but there is not restriction on how far apart they are from each other). It should be possible to adapt it in the case in which one of them vanishes by similar arguments to those in Desvillettes and Fellner (2007). This and the following Lemma are the main points in the paper where the positivity of the $d_{i}$ is used.

Using Lemma 3.1 in (23) one readily sees that

$$
\|h\|_{2}^{2} \leq e^{-2 \lambda_{*} t}\left\|h_{0}\right\|_{2}^{2}
$$

for any solution $h$ to (17)-(18) satisfying (19), where $h_{0}:=\left(h_{i, 0}\right)_{i=1, \ldots, 4}:=\left(a_{i, 0}-\right.$ $\left.a_{i, \infty}\right)_{i=1, \ldots, 4}$.

In fact, one can find a slightly different inequality that shows $\langle h, D h+L h\rangle$ actually bounds a stronger norm than $\|h\|_{2}$ :

Lemma 3.3. Take numbers $a_{i, \infty}>0$ for $i=1, \ldots, 4$. Assume Hypotheses 2.1 and 2.2, and define the linear operator $T$ by (21)-(22). There exists $\lambda>0$ such that

$$
\langle h, T h\rangle \leq-\lambda\|h\|_{2}^{2}-\lambda\|\nabla h\|_{2}^{2}
$$

for all $h \in\left(L^{2}(\Omega)\right)^{4}$ satisfying (19). The constant $\lambda$ depends only on the dimension $d$, the domain $\Omega$, the numbers $a_{i, \infty}, i=1, \ldots, 4$ and the diffusion constants $d_{i}, i=1, \ldots, 4$.

Proof. We set

$$
\tilde{L}_{i} h:=d_{i} \Delta h_{i}+L_{i} h-\frac{1}{2} d_{i} \Delta h_{i}=\frac{1}{2} d_{i} \Delta h_{i}+L_{i} h
$$

so that

$$
2\langle h, \tilde{L} h\rangle=-\sum_{i=1}^{4} \int_{\Omega} \frac{\left|\nabla h_{i}\right|^{2}}{a_{i, \infty}} \mathrm{d} x-2 C_{M} \int_{\Omega}\left(\frac{h_{1}}{a_{1, \infty}}+\frac{h_{3}}{a_{3, \infty}}-\frac{h_{2}}{a_{2, \infty}}-\frac{h_{4}}{a_{4, \infty}}\right)^{2} \mathrm{~d} x
$$

One sees that the inequality

$$
2\langle h, \tilde{L} h\rangle \leq-\tilde{\lambda}\|h\|_{2}^{2},
$$

still holds for some $\tilde{\lambda}>0$, so that

$$
2\langle h, T h\rangle \leq-\tilde{\lambda}\|h\|_{2}^{2}-\sum_{i=1}^{4} d_{i} \int_{\Omega} \frac{\left|\nabla h_{i}\right|^{2}}{a_{i, \infty}} \mathrm{d} x \leq-\tilde{\lambda}\|h\|_{2}^{2}-\min _{i=1, \ldots, 4}\left\{d_{i}\right\}\|\nabla h\|_{2}^{2} .
$$

We will use this strengthened inequality in the following section. 


\section{Local $L^{2}$ stability}

We now plan to use Lemma 3.3 in order to deduce $L^{\infty}\left([0, \infty), L^{2}(\Omega)\right)$ bounds on solutions to the nonlinear system (4)-(9), together with an exponential convergence to equilibrium of solutions:

Theorem 4.1. Assume Hypotheses 2.1, 2.2 and 2.3, and assume $d \leq 4$. Let $\left(a_{i}\right)_{i=1, \ldots, 4}$ be a solution to the system (8)-(9). Let $a_{i, \infty}, i=1, \ldots, 4$, denote the only positive equilibrium of (8) with the same invariants as $\left(a_{i, 0}\right)_{i=1, \ldots, 4}$ (that is, satisfying (12)).

There exist positive constants $\lambda, \epsilon>0$ depending on $d,\left(d_{i}\right)_{i=1, \ldots, 4},\left(a_{i, \infty}\right)_{i=1, \ldots, 4}$ and $\Omega$, such that

$$
\sum_{i=1}^{4} \int_{\Omega} \frac{\left|a_{i}(t, x)-a_{i, \infty}\right|^{2}}{a_{i, \infty}} \mathrm{d} x \leq e^{-\lambda t} \sum_{i=1}^{4} \int_{\Omega} \frac{\left|a_{i, 0}(x)-a_{i, \infty}\right|^{2}}{a_{i, \infty}} \mathrm{d} x
$$

whenever the $a_{i, 0}$ satisfy

$$
\sum_{i=1}^{4} \int_{\Omega} \frac{\left|a_{i, 0}(x)-a_{i, \infty}\right|^{2}}{a_{i, \infty}} \mathrm{d} x \leq \epsilon .
$$

Remark 4.2. All constants in the above statement can be explicitly given in terms of the parameters of the problem, as can be seen from the proof.

Remark 4.3. In particular we have $L^{1}$ convergence: $\sum_{i=1}^{4}\left\|a_{i}-a_{i, \infty}\right\|_{L^{1}(\Omega)}^{2} \leq K e^{-\lambda t}$ for some constant $K$ depending on the parameters of the problem and the initial data.

Proof of Theorem 4.1. In this proof we denote by $C, C_{1}, C_{2}, \ldots$ any number that depends only on the parameters in the statement $\left(d,\left(d_{i}\right)_{i=1, \ldots, 4},\left(a_{i, \infty}\right)_{i=1, \ldots, 4}\right.$ and $\left.\Omega\right)$. We deduce an a priori estimate on the $L^{2}$ norm of the solution, which can be rigorously justified by a standard approximation argument (for example by truncating the nonlinearity of the equation).

Consider a solution $\left(a_{1}, a_{2}, a_{3}, a_{4}\right)$ to the system (4)-(9). Defining $h_{i}$ as before,

$$
a_{i}=a_{i, \infty}+h_{i}, \quad i=1, \ldots, 4,
$$

we have that the difference to equilibrium $h=\left(h_{1}, h_{2}, h_{3}, h_{4}\right)$ satisfies (using the notation of Section 3)

$$
\left.\begin{array}{l}
\partial_{t} h_{1}=d_{1} \Delta h_{1}+L_{1} h-h_{1} h_{3}+h_{2} h_{4}, \\
\partial_{t} h_{2}=d_{2} \Delta h_{2}+L_{2} h+h_{1} h_{3}-h_{2} h_{4}, \\
\partial_{t} h_{3}=d_{3} \Delta h_{3}+L_{3} h-h_{1} h_{3}+h_{2} h_{4}, \\
\partial_{t} h_{4}=d_{4} \Delta h_{4}+L_{4} h+h_{1} h_{3}-h_{2} h_{4},
\end{array}\right\} \quad t \geq 0, x \in \Omega
$$

together with the mass-conserving boundary conditions (18). Since the solution $\left(a_{i}\right)_{i=1, \ldots, 4}$ satisfies the conservation laws (12), the $h_{i}$ satisfy (19). If we denote the nonlinear terms in the right hand side of (28) by

$$
N(h)=\left(N_{1} h, N_{2} h, N_{3} h, N_{4} h\right)
$$

we may write (28) as

$$
\partial_{t} h=D h+L h+N(h)=T h+N(h) .
$$


We can now estimate the evolution of the $L^{2}$ norm defined in (6) by using Lemma 3.3:

$$
\frac{\mathrm{d}}{\mathrm{d} t}\|h\|_{2}^{2}=2\langle h, T h\rangle+2\langle h, N(h)\rangle \leq-2 \lambda\|h\|_{2}^{2}-2 \lambda\|\nabla h\|_{2}^{2}+2\langle h, N(h)\rangle .
$$

We use the Gagliardo-Nirenberg inequality in Lemma 2.3 to obtain

$$
\|h\|_{3} \leq C_{1}\|\nabla h\|_{2}^{\theta}\|h\|_{2}^{1-\theta}+C_{1}\|h\|_{2}, \quad \theta=\frac{d}{6}
$$

(which is actually valid for $d<6$ ). This implies

$$
\|h\|_{3}^{\frac{2}{\theta}} \leq C_{2}\|\nabla h\|_{2}^{2}\|h\|_{2}^{\frac{2(1-\theta)}{\theta}}+C_{2}\|h\|_{2}^{\frac{2}{\theta}}
$$

that is,

$$
-2 \lambda\|\nabla h\|_{2}^{2} \leq-\lambda\|\nabla h\|_{2}^{2} \leq-\frac{\lambda}{C_{2}}\|h\|_{3}^{\frac{2}{\theta}}\|h\|_{2}^{-\frac{2(1-\theta)}{\theta}}+\lambda\|h\|_{2}^{2} .
$$

In order to show that the term $\langle h, N(h)\rangle$ is negligible close to equilibrium we need to estimate quantities of the type $\int_{\Omega} h_{i} h_{j} h_{k} \mathrm{~d} x$, with $i, j, k \in\{1,2,3,4\}$. We do that by Hölder's inequality:

$$
\left|\int_{\Omega} h_{i} h_{j} h_{k} \mathrm{~d} x\right| \leq C_{3}\|h\|_{3}^{3}
$$

for a certain constant $C_{3}$ depending only on the equilibrium values $\left(a_{i, \infty}\right)_{i=1, \ldots, 4}$. Consequently,

$$
\langle h, N(h)\rangle \leq C_{4}\|h\|_{3}^{3} .
$$

Using (30) and (32) in (29),

$$
\begin{aligned}
\frac{\mathrm{d}}{\mathrm{d} t}\|h\|_{2}^{2} & \leq-\lambda\|h\|_{2}^{2}-\frac{\lambda}{C_{2}}\|h\|_{3}^{\frac{12}{d}}\|h\|_{2}^{\frac{2 d-12}{d}}+2 C_{4}\|h\|_{3}^{3} \\
& =-\lambda\|h\|_{2}^{2}-\|h\|_{3}^{\frac{12}{d}}\left(\frac{\lambda}{C_{2}}\|h\|_{2}^{\frac{2 d-12}{d}}-2 C_{4}\|h\|_{3}^{\frac{3 d-12}{d}}\right) \\
& \leq-\lambda\|h\|_{2}^{2}-\|h\|_{3}^{\frac{12}{d}}\left(\frac{\lambda}{C_{2}}\|h\|_{2}^{\frac{2 d-12}{d}}-C_{5}\|h\|_{2}^{\frac{3 d-12}{d}}\right) \\
& =-\lambda\|h\|_{2}^{2}-\|h\|_{3}^{\frac{12}{d}}\|h\|_{2}^{\frac{2 d-12}{d}}\left(\frac{\lambda}{C_{2}}-C_{5}\|h\|_{2}\right),
\end{aligned}
$$

where in the second inequality we used that $3 d-12 \leq 0$ and $\|h\|_{2} \leq C\|h\|_{3}$ for some constant $C$. If it is initially true that

$$
\left\|h_{0}\right\|_{2} \leq \frac{\lambda}{C_{2} C_{5}}
$$

then $\|h\|_{2}$ is decreasing in time and we obtain the result.

Remark 4.4. One may wonder if choosing a norm $\|h\|_{p}$ different from $\|h\|_{3}$ may give an improvement in the above argument, perhaps making it work in dimensions $d \geq 4$. The authors have tried this with other choices of $p$, and in particular for $p=2_{*}=(2 d) /(d-2)$ the Poincaré dual of 2 . The dimension $d=4$ seems to be a limitation of the argument and not of the specific exponents used in the proof. 


\section{$5 \quad$ Regularity of close-to-equilibrium solutions}

We first extend the $L^{2}$ estimates of the previous section to $L^{p}$ estimates for a certain range of $p>2$.

Proposition 5.1. Assume Hypotheses 2.1, 2.2 and 2.3, and let $d \leq 4$. Let $\left(a_{i}\right)_{i=1, \ldots, 4}$ be a solution to the system (8)-(9). Let $a_{i, \infty}, i=1, \ldots, 4$, denote the only positive equilibrium of (4) with the same invariants as $\left(a_{i, 0}\right)_{i=1, \ldots, 4}$ (that is, satisfying (12)).

Assume also that $\left\|a_{i, 0}\right\|_{p}<\infty$ for some $2 \leq p<\infty$. Then there exist positive constants $\lambda, K, \epsilon>0$ depending on $p, d,\left(d_{i}\right)_{i=1, \ldots, 4},\left(a_{i, \infty}\right)_{i=1, \ldots, 4},\left\|a_{0}\right\|_{p}$ and $\Omega$, such that

$$
\sum_{i=1}^{4} \int_{\Omega} \frac{\left|a_{i}(t, x)-a_{i, \infty}\right|^{p}}{a_{i, \infty}} \mathrm{d} x \leq K(1+t)
$$

whenever the $a_{i, 0}$ satisfy

$$
\sum_{i=1}^{4} \int_{\Omega} \frac{\left|a_{i, 0}(x)-a_{i, \infty}\right|^{2}}{a_{i, \infty}} \mathrm{d} x \leq \epsilon .
$$

Proof. For $d=1$ the result is given directly by Lemma 2.6 (the time dependence can be checked to be less than $1+t)$. For $d \geq 2$ and any $p>1$ we compute

$$
\begin{aligned}
\frac{\mathrm{d}}{\mathrm{d} t}\|h\|_{p}^{p} & =\frac{\mathrm{d}}{\mathrm{d} t} \sum_{i=1}^{4} \int_{\Omega} \frac{\left|h_{i}\right|^{p}}{a_{i, \infty}} \mathrm{d} t \\
& =-\frac{p-1}{4 p} \sum_{i=1}^{4} d_{i} \int_{\Omega} \frac{\left|\nabla h_{i}^{p / 2}\right|^{2}}{a_{i, \infty}} \mathrm{d} x \\
& +p \sum_{i=1}^{4} \frac{1}{a_{i, \infty}} \int_{\Omega} \operatorname{sign}\left(h_{i}\right)\left|h_{i}\right|^{p-1}\left(L_{i}(h)+N_{i}(h)\right) \mathrm{d} x .
\end{aligned}
$$

Using that for any $i, j=1, \ldots, 4$

$$
\int_{\Omega}\left|h_{i}\right|^{p-1}\left|h_{j}\right| d x \leq C_{1}\|h\|_{p}^{p}
$$

the linear term corresponding to $L$ is controlled by the $L^{p}$ norm:

$$
p \sum_{i=1}^{4} \frac{1}{a_{i, \infty}} \int_{\Omega} \operatorname{sign}\left(h_{i}\right)\left|h_{i}\right|^{p-1} L_{i}(h) d x \leq C_{L}\|h\|_{p}^{p} .
$$

The nonlinear term can be bounded by an application of Hölder's inquality similar to the one in the previous section, as

$$
\int_{\Omega}\left|h_{i}\right|\left|h_{j}\right|\left|h_{k}\right|^{p-1} d x \leq C_{2}\|h\|_{p+1}^{p+1}
$$

for any $i, j, k \in\{1,2,3,4\}$, obtaining

$$
p \sum_{i=1}^{4} \frac{1}{a_{i, \infty}} \int_{\Omega} \operatorname{sign}\left(h_{i}\right)\left|h_{i}\right|^{p-1} N_{i}(h) d x \leq C_{N}\|h\|_{p+1}^{p+1} .
$$


Using (34) and (35) in (33) we have

$$
\frac{\mathrm{d}}{\mathrm{d} t}\|h\|_{p}^{p} \leq-\frac{p-1}{4 p} \sum_{i=1}^{4} d_{i} \int_{\Omega} \frac{\left|\nabla h_{i}^{p / 2}\right|^{2}}{a_{i, \infty}} \mathrm{d} x+C_{L}\|h\|_{p}^{p}+C_{N}\|h\|_{p+1}^{p+1} .
$$

Proof for $d=3,4$. In dimensions $d>2$ the diffusion term can be bounded as follows using the Gagliardo-Nirenberg inequality (14):

$$
-\frac{p-1}{4 p} \sum_{i=1}^{4} d_{i} \int_{\Omega} \frac{\left|\nabla h_{i}^{p / 2}\right|^{2}}{a_{i, \infty}} \mathrm{d} x \leq-C_{D}\|h\|_{q}^{p}+C_{D}\|h\|_{p}^{p},
$$

where $q:=\frac{d p}{d-2}$ (notice that the minimum of the diffusion coefficients $d_{i}$ appears implicitly in the constant $C_{D}$ ). Using (37) in (36) we have

$$
\frac{\mathrm{d}}{\mathrm{d} t}\|h\|_{p}^{p} \leq-C_{D}\|h\|_{q}^{p}+C_{3}\|h\|_{p}^{p}+C_{N}\|h\|_{p+1}^{p+1} .
$$

We now use the interpolation

$$
\|h\|_{p} \leq\|h\|_{1}^{1-\mu}\|h\|_{q}^{\mu}
$$

with

$$
\mu=\frac{q(1-p)}{p(1-q)}=\frac{d(1-p)}{d(1-p)-2}
$$

together with the fact that the $L^{1}$ norm of $h$ is uniformly bounded in time (since the total mass is conserved), to obtain

$$
\|h\|_{p} \leq C_{2}\|h\|_{q}^{\mu}
$$

This gives

$$
-C_{D}\|h\|_{q}^{p} \leq-C_{4}\|h\|_{p}^{p / \mu}
$$

To control the nonlinear part we may use interpolation, noticing that $2 \leq p+1 \leq q$ (which holds since $\frac{d-2}{2} \leq p$ )

$$
\|h\|_{p+1}^{p+1} \leq\|h\|_{2}^{\theta(p+1)}\|h\|_{q}^{(p+1)(1-\theta)}
$$

where $\theta=\frac{2(q-p-1)}{(q-2)(p+1)}$. In dimension $d \leq 4$ the exponent of $\|h\|_{q}$ is smaller than $p$, since in this case $2 p-q \leq 0$ and thus

$$
(p+1)(1-\theta)-p=1-\theta(p+1)=1-\frac{2(q-p-1)}{q-2}=\frac{2 p-q}{q-2} \leq 0 .
$$

Since $p \leq q$ and the domain $\Omega$ is bounded, we have

$$
\|h\|_{q}^{(p+1)(1-\theta)} \leq C_{5}\|h\|_{q}^{p}\|h\|_{2}^{(p+1)(1-\theta)-p}
$$

and hence from (40) one obtains

$$
\|h\|_{p+1}^{p+1} \leq C_{5}\|h\|_{2}\|h\|_{q}^{p}
$$


Finally, using (39) and (41) in (38), breaking the term $-C_{D}\|h\|_{q}^{p}$ into two halves, we have

$$
\begin{aligned}
\frac{\mathrm{d}}{\mathrm{d} t}\|h\|_{p}^{p} \leq-\frac{1}{2} C_{D}\|h\|_{q}^{p}-\frac{1}{2} C_{D}\|h\|_{q}^{p}+C_{3}\|h\|_{p}^{p}+C_{N}\|h\|_{p+1}^{p+1} . \\
=-\frac{1}{2} C_{D}\|h\|_{q}^{p}-\frac{1}{2} C_{4}\|h\|_{p}^{p / \mu}+C_{3}\|h\|_{p}^{p}+C_{N} C_{5}\|h\|_{2}\|h\|_{q}^{p} \\
=-\frac{1}{2} C_{4}\|h\|_{p}^{p / \mu}+C_{3}\|h\|_{p}^{p}-\|h\|_{q}^{p}\left(\frac{1}{2} C_{D}-C_{N} C_{5}\|h\|_{2}\right) .
\end{aligned}
$$

If the initial condition satisfies

$$
\left\|h_{0}\right\|_{2} \leq \frac{C_{D}}{2 C_{N} C_{5}}
$$

then this is also the case for any $t \geq 0$, and the last term in (42) is nonpositive. This shows that

$$
\frac{\mathrm{d}}{\mathrm{d} t}\|h\|_{p}^{p} \leq-\frac{1}{2} C_{4}\|h\|_{p}^{p / \mu}+C_{3}\|h\|_{p}^{p} .
$$

Since $\mu<1$, this differential inequality for $\|h\|_{p}^{p}$ shows that $\|h\|_{p}$ is bounded uniformly in time by a certain constant $K$ which may depend also on $\left\|h_{0}\right\|_{p}$. (Notice that the $(1+t)$ factor is not needed in this case).

Proof for $d=2$. Take any $2<p<4$, and choose $s, q$ with $1<s<\frac{2 q}{p}$. Using the Gagliardo-Nirenberg-Sobolev inequality we have

$$
\|h\|_{q}^{\frac{p}{2}}=\left\|h^{\frac{p}{2}}\right\|_{\frac{2 q}{p}} \leq C\left\|\nabla h^{\frac{p}{2}}\right\|_{2}^{\theta}\left\|h^{\frac{p}{2}}\right\|_{s}^{1-\theta}+C\left\|h^{\frac{p}{2}}\right\|_{1} \leq C\left\|\nabla h^{\frac{p}{2}}\right\|_{2}^{\theta}\|h\|_{\frac{p s}{2}}^{\frac{p(1-\theta)}{2}}+C\|h\|_{p}^{\frac{p}{2}}
$$

with

$$
\frac{p}{2 q}=\theta \frac{d-2}{2 d}+\frac{1-\theta}{s}
$$

Rearranging the terms and choosing $s=4 / p>1$ (which implies $q>2$ ) we have

$$
-\left\|\nabla h^{\frac{p}{2}}\right\|_{2}^{2} \leq-C_{1}\|h\|_{2}^{\frac{-p(1-\theta)}{\theta}}\|h\|_{q}^{\frac{p}{\theta}}+\|h\|_{p}^{p}
$$

with

$$
\theta=\frac{d p(q-2)}{q(2(2-d)+d p)}
$$

We will use this bound on the diffusive term in (36). In order to control the $\|h\|_{p}^{p}$ term in (36) we further bound the diffusive term as follows: by interpolation,

$$
\|h\|_{p} \leq\|h\|_{1}^{\frac{q-p}{p(q-1)}}\|h\|_{q}^{\frac{q(p-1)}{p(q-1)}} \leq C_{2}\|h\|_{q}^{\frac{q(p-1)}{p(q-1)}}
$$

where the last inequality is due to the boundedness of $\|h\|_{1}$. Using this in (43) we have

$$
\left\|\nabla h^{\frac{p}{2}}\right\|_{2}^{2} \geq C_{3}\|h\|_{2}^{\frac{-p(1-\theta)}{\theta}}\|h\|_{p}^{\frac{p}{\theta} \frac{p(q-1)}{q(p-1)}} \geq C_{4}\|h\|_{p}^{\frac{p}{\theta} \frac{p(q-1)}{q(p-1)}}-\|h\|_{p}^{p},
$$

now due to the boundedness of $\|h\|_{2}$.

In order to control the nonlinear term we interpolate in a similar way as before, with any $q>p+1$ :

$$
\|h\|_{p+1}^{p+1} \leq\|h\|_{2}^{\frac{2(q-(p+1))}{q-2}}\|h\|_{q}^{\frac{p}{\theta}}\|h\|_{q}^{\frac{q(p-1)}{q-2}-\frac{p}{\theta}} .
$$


Noticing that the exponent of the last term is nonpositive for $d \leq 4$, and that $\|h\|_{2}$ is bounded by a constant times $\|h\|_{q}$, we have

$$
\|h\|_{p+1}^{p+1} \leq C_{5}\|h\|_{2}^{p+1-\frac{p}{\theta}}\|h\|_{q}^{\frac{p}{\theta}}
$$

Now we use (43), (44) and (45) in (36), breaking again the diffusive term into two halves:

$$
\begin{aligned}
& \frac{\mathrm{d}}{\mathrm{d} t}\|h\|_{p}^{p} \leq-C_{6}\left\|\nabla h^{\frac{p}{2}}\right\|_{2}^{2}+C_{10}\|h\|_{p}^{p}+C_{N}\|h\|_{p+1}^{p+1} \\
& \leq-C_{7}\|h\|_{2}^{\frac{-p(1-\theta)}{\theta}}\|h\|_{q}^{\frac{p}{\theta}}-C_{8}\|h\|_{p}^{\frac{p}{\theta} \frac{p(q-1)}{q(p-1)}}+C_{10}\|h\|_{p}^{p}+C_{9}\|h\|_{2}^{p+1-\frac{p}{\theta}}\|h\|_{q}^{\frac{p}{\theta}} \cdot \\
& \quad=-C_{8}\|h\|_{p}^{\frac{p}{\theta} \frac{p(q-1)}{q(p-1)}}+C_{10}\|h\|_{p}^{p}-\|h\|_{q}^{\frac{p}{\theta}}\left(C_{7}\|h\|_{2}^{\frac{-p(1-\theta)}{\theta}}-C_{9}\|h\|_{2}^{p+1-\frac{p}{\theta}}\right)
\end{aligned}
$$

The term in parentheses is nonnegative when

$$
\|h\|_{2}^{\frac{p(1-\theta)}{\theta}+p+1-\frac{p}{\theta}}=\|h\|_{2} \leq \frac{C_{7}}{C_{9}}
$$

which holds for all $t \geq 0$ as long as it holds for the initial condition, since $\|h\|_{2}$ is nonincreasing in time. Assuming this we finally have

$$
\frac{\mathrm{d}}{\mathrm{d} t}\|h\|_{p}^{p} \leq-C_{8}\|h\|_{p}^{\frac{p}{\theta} \frac{p(q-1)}{q(p-1)}}+C_{10}\|h\|_{p}^{p}
$$

Using that $\theta \leq \frac{p(q-1)}{q(p-1)}$, we see that this differential inequality shows that $\|h\|_{p}$ is uniformly bounded in time. Since this is valid for $2 \leq p<4$, this shows that all the nonlinear terms in (28) are uniformly bounded in $L^{s}(\Omega)$ for $1 \leq s<2$ (and of course the linear ones are as well). Lemma 2.6 gives the result for all $p$.

We are finally in position to complete the proof of Theorem 2.1:

Proof of Theorem 2.1. Theorem 4.1 and Proposition 5.1 give the $L^{p}$ decay since

$$
\|h\|_{p} \leq\|h\|_{2}^{\theta}\|h\|_{p+1}^{1-\theta} \leq C\|h\|_{2}^{\theta} \leq \tilde{C} e^{-\lambda \theta t} .
$$

If we assume that $a_{i, 0} \in L^{\infty}(\Omega)$ for $i=1, \ldots, 4$ then this implies that all the nonlinear terms of equation $(8)$ are in $L^{p}(\Omega)$, for all $1 \leq p<\infty$. Lemma 2.6 then shows that the $a_{i}$ are bounded in $L^{\infty}(\Omega)$ (using the decay of the $L^{p}$ norms, one may check the proof to see that the $L^{\infty}$ bound does not depend on time). Classical bootstrap arguments then show that the solution $\left(a_{i}\right)_{i=1, \ldots, 4}$ is in fact infinitely differentiable for $t>0$.

\section{General quadratic systems with detailed balance}

The arguments in the previous sections can be adapted to any reaction-diffusion system with at most quadratic nonlinearities, with the caveat that the constant $\lambda$ giving the speed of convergence in Theorem 2.1 cannot be estimated in a constructive way. One can however show that the linearised system still has a positive spectral gap (with no estimate on its size) and relate the speed $\lambda$ to the size of this spectral gap. 


\subsection{General setting and main result}

Let us first describe the setting in which our result holds. We consider a system of $I \geq 2$ species, denoted $A_{1}, \ldots, A_{I}$, undergoing a number $R \geq 1$ of different reactions:

$$
\alpha_{1}^{r} A_{1}+\cdots+\alpha_{N}^{r} A_{I} \underset{k_{b}^{r}}{\stackrel{k_{f}^{r}}{\rightleftharpoons}} \beta_{1}^{r} A_{1}+\cdots+\beta_{N}^{r} A_{I}, \quad r=1, \ldots, R .
$$

The description of these processes requires somewhat heavy notation, which makes clear the reason why we have chosen to present our results first for the four-species system. We follow the clear presentation given in Fellner and Tang (2017). The positive numbers $k_{f}^{r}$ and $k_{b}^{r}$, for $r=1 \ldots, R$, denote the forward and backward reaction rates, respectively, for each of the $R$ reactions. The vectors $\alpha^{r}=\left(\alpha_{1}^{r}, \ldots, \alpha_{I}^{r}\right)$ and $\beta^{r}=\left(\beta_{1}^{r}, \ldots, \beta_{I}^{r}\right)$ are the stoichiometric coefficients which specify the number of particles of each species that take part in each reaction. Let us denote by $a_{i}=a_{i}(t, x)$ the concentration of the species $i$ at time $t$ and position $x \in \Omega$ (for $i=1, \ldots, I$ ). If the diffusion coefficient of the species $A_{i}$ is $d_{i}>0$ then the evolution equation that describes the concentrations $a=\left(a_{i}\right)_{i=1, \ldots, I}$ is

$$
\partial_{t} a_{i}=d_{i} \Delta a_{i}-R_{i}(a), \quad i=1, \ldots, I
$$

with no-flux boundary conditions as before,

$$
\nabla_{x} a_{i}(t, x) \cdot \nu(x)=0, \quad t>0, x \in \partial \Omega, i=1, \ldots, I,
$$

where $\mathbf{R}(a)=\left(R_{1}(a), \ldots, R_{I}(a)\right)$ is given by

$$
\mathbf{R}(a)=\sum_{r=1}^{R}\left(k_{f}^{r} a^{\alpha^{r}}-k_{b}^{r} a^{\beta^{r}}\right)\left(\alpha^{r}-\beta^{r}\right) .
$$

Here we use the multiindex notation to write

$$
a^{\alpha}=\prod_{i=1}^{I} a_{i}^{\alpha_{i}}, \quad r=1, \ldots, R,
$$

for any vector $\alpha$ with $I$ components. We also set an initial condition for the $a_{i}$ :

$$
a_{i}(0, x)=a_{i}^{0}(x), \quad x \in \Omega, \quad i=1, \ldots, I .
$$

One can find the conserved quantities of this system as follows: define the $R \times I$ matrix $W$ by writing the vectors $\beta^{r}-\alpha^{r}$ as rows:

$$
W:=\left(\begin{array}{c}
\beta^{1}-\alpha^{1} \\
\vdots \\
\beta^{R}-\alpha^{R}
\end{array}\right)
$$

and take a matrix $\mathbb{Q}$ whose rows are a basis of $\left(\operatorname{Im} W^{\top}\right)^{\top}=\operatorname{Ker} W$ (the orthogonal complement of the image of $W^{\top}$ in the usual scalar product or, equivalently, the kernel of $W$ ). This ensures that $\mathbb{Q} W^{\top}=0$, and since we can write

$$
\mathbf{R}(a)=W^{\top} \mathbf{K}(a),
$$


where $\mathbf{K}(a):=\left(k_{f}^{r} a^{\alpha^{r}}-k_{b}^{r} a^{\beta^{r}}\right)_{r=1, \ldots, R}$, we see that the vector

$$
\int_{\Omega} \mathbb{Q} a(t, x) \mathrm{d} x
$$

is conserved along the evolution (47) (see Fellner and Tang (2017) for details). An equilibrium of the system (47) is a vector $a_{\infty}=\left(a_{1, \infty}, \ldots, a_{I, \infty}\right)$ with nonnegative entries such that $\mathbf{R}\left(a_{\infty}\right)=0$. A detailed balance equilibrium of (47) is a vector $a_{\infty}=$ $\left(a_{1, \infty}, \ldots, a_{I, \infty}\right)$ with strictly positive entries such that

$$
k_{f}^{r} a_{\infty}^{\alpha^{r}}-k_{b}^{r} a_{\infty}^{\beta^{r}}=0 \quad \text { for all } r=1, \ldots, R .
$$

For illustration, the case of the four-species system is included in this setting: it corresponds to $R=1, I=4, \alpha^{1}=(1,0,1,0), \beta^{1}=(0,1,0,1)$,

$$
W=(-1,1,-1,1)
$$

and we may choose

$$
\mathbb{Q}=\left(\begin{array}{llll}
1 & 1 & 0 & 0 \\
1 & 0 & 0 & 1 \\
0 & 1 & 1 & 0
\end{array}\right)
$$

which corresponds to the conserved quantities $M_{12}, M_{14}$ and $M_{23}$ as described in Section 2. Since in this case there is only one pair of reactions taking place, every equilibrium with positive entries is a detailed balance equilibrium.

Let us describe our assumptions. Our first set mimics the conditions we used for the four-species system: we always assume Hypothesis 2.1 on the domain $\Omega$ and the following positivity conditions on the parameters:

Hypothesis 6.1 (Positivity of the constants). $I \geq 2$ and $R \geq 1$ are integers, and the diffusion rates $d_{1}, \ldots, d_{I}$ are strictly positive numbers. The reaction constants $k_{f}^{r}, k_{b}^{r}$ are strictly positive numbers for $r=1, \ldots, R$. For $r=1, \ldots, R$ the vectors $\alpha^{r}, \beta^{r} \in \mathbb{R}^{I}$ have nonnegative integer coordinates.

We always need to assume that the system has at most quadratic nonlinearities; that is, that each of the reactions happens with at most two reactants:

Hypothesis 6.2 (The system is at most quadratic). The sum of the coordinates of each of the vectors $\alpha^{r}, \beta^{r} \in \mathbb{R}^{I}, r=1, \ldots, R$, is less or equal than 2 . That is,

$$
\sum_{i=1}^{I} \alpha_{i}^{r} \leq 2, \quad \sum_{i=1}^{I} \beta_{i}^{r} \leq 2, \quad \text { for } r=1, \ldots, R .
$$

The previous hypothesis is just a way of saying that each reaction must have either (a) one reactant, so the resulting term in $\mathbf{R}(a)$ is linear, or (b) two reactants, in which the resulting term is quadratic. These two reactants could be the same one, so one of the components of $\alpha^{r}$ or $\beta^{r}$ can be 2 (and in that case the rest of them must be 0 ).

Given the vectors $\alpha^{r}, \beta^{r}$ for $r=1, \ldots, R$ we can define a matrix $\mathbb{Q}$ of "conserved quantities" as described at the beginning of this section. We fix such a matrix $\mathbb{Q}$ for the rest of this paper. The following assumption corresponds to Hypothesis 2.3 in this general setting: it says that all conserved quantities corresponding to the initial data are strictly positive: 
Hypothesis 6.3 (Positivity of the initial condition). The functions $a_{1,0}, \ldots, a_{I, 0}: \Omega \rightarrow$ $\mathbb{R}$ are in $L^{2}(\Omega)$, are nonnegative, and satisfy that

$$
\int_{\Omega} \mathbb{Q} a_{0}(x) \mathrm{d} x \text { has strictly positive components, }
$$

where $a_{0}:=\left(a_{1,0}, \ldots, a_{I, 0}\right)$.

Finally a crucial assumption is that the system associated to the reactions (46) satisfies the detailed balance condition:

Hypothesis 6.4 (Detailed balance). The system (47) has a detailed balance equilibrium $a_{\infty}=\left(a_{1, \infty}, \ldots, a_{I, \infty}\right)$ satisfying the condition that

$$
\mathbb{Q} a_{\infty}=\int_{\Omega} \mathbb{Q} a_{0}(x) \mathrm{d} x .
$$

(That is, such that $\int_{\Omega}\left(a_{\infty}-a_{0}\right)$ is in the image of $\left.W^{\top}\right)$.

To avoid confusion: we are assuming that there is a detailed balance equilibrium (one whose components are strictly positive and satisfy (50)), but we are not assuming it must be the only one. In particular, we are not ruling out the existence of "boundary equilibria", that is, equilibria with some components equal to 0 (satisfying (51)). These boundary equilibria are an important obstacle in the study of the global behaviour of reaction equations in general (even without diffusion) and are closely linked to the global attractor conjecture (Horn and Jackson, 1972). Some results on reaction-diffusion systems with boundary equilibria have been recently investigated in Desvillettes et al. (2017). Our statement in this general setting is that solutions in dimension $d \leq 4$ which are close to a detailed balance equilibria are regular (classical) and relax exponentially to equilibrium. In other words, detailed balance equilibria are always locally asymptotically stable, at least in dimension 4 and below:

Theorem 6.1. Assume Hypothesis 2.1 and Hypotheses 6.1-6.4, and let $d \leq 4$. Let $\left(a_{i}\right)_{i=1, \ldots, I}$ be a solution to the system $(47)-(49)$. Let $a_{i, \infty}, i=1, \ldots, I$, denote a detailed balance equilibrium of (47) with the same invariants as $\left(a_{i, 0}\right)_{i=1, \ldots, I}$ (that is, satisfying (51)).

Then for any $2 \leq p<\infty$ there exist positive constants $\lambda, K, \epsilon>0$ depending on $p$, $d,\left(d_{i}\right)_{i=1, \ldots, I},\left(a_{i, \infty}\right)_{i=1, \ldots, I}, \Omega$ and $\left\|a_{0}\right\|_{p}$ such that

$$
\sum_{i=1}^{I} \int_{\Omega} \frac{\left|a_{i}(t, x)-a_{i, \infty}\right|^{p}}{a_{i, \infty}} \mathrm{d} x \leq K e^{-\lambda t}
$$

whenever the $a_{i, 0}$ satisfy

$$
\sum_{i=1}^{I} \int_{\Omega} \frac{\left|a_{i, 0}(x)-a_{i, \infty}\right|^{2}}{a_{i, \infty}} \mathrm{d} x \leq \epsilon .
$$

As a consequence, if $a_{i, 0} \in L^{\infty}(\Omega)$, then the solution to (47)-(49) is uniformly bounded in $L^{\infty}(\Omega)$, and is a classical solution for $t>0$. 
One important difference with Theorem 2.1 is that in this generality the constant $\lambda$ cannot be estimated in any constructive way. We are able to prove that there exists $\lambda$ which satisfies the theorem, but no explicit way is given to estimate the value of $\lambda$ based on the parameters of the problem. On the other hand, one cannot expect an estimate in full generality. It should be noted that the system (47) contains as a particular case the Kolmogorov forward equations for a discrete Markov process with jump rates $k_{f}^{r}, k_{b}^{r}$. (This corresponds to the case of homogeneous solutions, with reactions where $\alpha^{r}$ and $\beta^{r}$ have only one nonzero entry, and this entry is equal to 1, yielding a linear equation). Estimating the speed of convergence to equilibrium for detailed balance discrete Markov processes is a broad problem in its own right and it is out of the scope of this paper.

The rest of this section contains the proof of Theorem 6.1, and we always assume the hypotheses of the theorem. The first observation towards it is to notice that the entropy structure is maintained by the detailed balance condition: using (50) one sees that a (regular) solution to (47)-(48) satisfies

$$
\begin{aligned}
& \frac{\mathrm{d}}{\mathrm{d} t} \sum_{i=1}^{I} \int_{\Omega} a_{i, \infty}\left(u_{i} \log u_{i}-u_{i}+1\right) \mathrm{d} x \\
& \quad=-\sum_{i=1}^{I} \int_{\Omega} d_{i} \frac{\left|\nabla a_{i}\right|^{2}}{a_{i}} \mathrm{~d} x-\sum_{r=1}^{R} \int_{\Omega} k_{f}^{r} a_{\infty}^{\alpha^{r}}\left(u^{\alpha^{r}}-u^{\beta^{r}}\right)\left(\log u^{\alpha^{r}}-\log u^{\beta^{r}}\right) \mathrm{d} x,
\end{aligned}
$$

where we call $u:=\left(u_{1}, \ldots, u_{I}\right)$ with

$$
u_{i} \equiv u_{i}(t, x):=\frac{a_{i}(t, x)}{a_{i, \infty}} \quad \text { for } i=1, \ldots, I .
$$

We follow the same steps as for the four-species system, indicating only the differences where the arguments are the same.

\subsection{The linearised system}

Considering the linearisation of (47) around the equilibrium $a_{\infty}=\left(a_{i, \infty}\right)_{i=1, \ldots, I}$ as in Section 3 at first order we obtain the following evolution equation for the perturbation $h=\left(h_{i}\right)_{i=1, \ldots, I}$ (defined so that $a_{i}=a_{i, \infty}+h_{i}$ for all $\left.i\right)$ :

$$
\partial_{t} h_{i}=d_{i} \Delta h_{i}+L_{i} h
$$

still with no-flux boundary conditions

$$
\nabla_{x} h_{i}(t, x) \cdot \nu(x)=0, \quad t>0, x \in \partial \Omega, i=1, \ldots, I,
$$

where the linear operator $L h=\left(L_{i} h\right)_{i=1, \ldots, I}$ is given by

$$
L_{i} h=-\sum_{j=1}^{I} \sum_{r=1}^{R} k_{f}^{r} a_{\infty}^{\alpha^{r}}\left(\alpha_{j}^{r}-\beta_{j}^{r}\right)\left(\alpha_{i}^{r}-\beta_{i}^{r}\right) \frac{h_{j}}{a_{j, \infty}}, \quad i=1, \ldots, I
$$

or, using matrix notation,

$$
L h=-\sum_{r=1}^{R} k_{f}^{r} a_{\infty}^{\alpha^{r}}\left(\alpha^{r}-\beta^{r}\right)^{\top}\left(\alpha^{r}-\beta^{r}\right) v
$$


with

$$
v \equiv\left(v_{1}, \ldots, v_{i}\right):=\left(\frac{h_{1}}{a_{1, \infty}}, \ldots, \frac{h_{I}}{a_{I, \infty}}\right)
$$

We can write (53) as

$$
\partial_{t} h=D h+L h=: T h,
$$

where $D h=\left(d_{i} \Delta h_{i}\right)_{i=1, \ldots, I}$. Due to the expression of $L$ it is clear that $\mathbb{Q} L h=0$ for all $h$, so solutions to (53)-(54) satisfy the same conservation laws as (47)-(48):

$$
\int_{\Omega} \mathbb{Q} h(t, x) \mathrm{d} x=\int_{\Omega} \mathbb{Q} h(0, x) \mathrm{d} x \quad \text { for all } t \geq 0 .
$$

This linearised system "inherits" an entropy structure from (52). Namely,

$$
\begin{aligned}
\frac{\mathrm{d}}{\mathrm{d} t} \sum_{i=1}^{I} \int_{\Omega} \frac{1}{a_{i, \infty}} h_{i}^{2} & =2 \sum_{i=1}^{I} \int_{\Omega} \frac{1}{a_{i, \infty}} h_{i} T_{i} h \\
& =-2 \sum_{i=1}^{I} d_{i} \int_{\Omega} \frac{\left|\nabla h_{i}\right|^{2}}{a_{i, \infty}} \mathrm{d} x-2 \sum_{r=1}^{R} \int_{\Omega} k_{f}^{r} a_{\infty}^{\alpha^{r}}\left(\sum_{i=1}^{I}\left(\alpha_{i}^{r}-\beta_{i}^{r}\right) \frac{h_{i}}{a_{i, \infty}}\right)^{2} \mathrm{~d} x .
\end{aligned}
$$

Disregarding for the moment the spatial dependence, we may consider the operator $L$ defined on the vector space $\mathbb{R}^{I}$. Since $\mathbb{Q} L h=0$ for all $h$, we may restrict $L$ to the vector space $X=\operatorname{Im} W^{\top}$. Our first result is that the linear operator $L$ has negative spectrum in this space (and as an immediate consequence, detailed balance equilibria are always isolated). This is known since at least Horn and Jackson (1972), but we prove it here for completeness:

Lemma 6.2. Assume the conditions of Theorem 6.1. There exists a constant $\lambda>0$ such that the spectrum of the linear operator $L: \operatorname{Im} W^{\top} \rightarrow \operatorname{Im} W^{\top}$ defined by (55) is contained in $(-\infty,-\lambda)$.

Proof. One sees from (55) that the matrix of $L$ is symmetric in the scalar product associated to the norm

$$
\|h\|^{2}:=\sum_{i=1}^{I} \frac{1}{a_{i, \infty}} h_{i}^{2} .
$$

Denote this inner product by $\langle\cdot, \cdot\rangle$. We emphasise that in this proof $\|\cdot\|$ and $\langle\cdot, \cdot\rangle$ denote a norm and inner product in $\mathbb{R}^{I}$, while in the rest of the section they denote a norm in $\left(L^{2}(\Omega)\right)^{I}$ (that is, with an additional integral in $\Omega$ ). Due the symmetry of $L$ in this inner product, all eigenvalues of $L$ are real. From the identity

$$
\begin{aligned}
\langle L h, h\rangle=\sum_{i=1}^{I} \frac{1}{a_{i, \infty}} h_{i} L_{i} h=-\sum_{r=1}^{R} k_{f}^{r} a_{\infty}^{\alpha^{r}}\left(\sum_{i=1}^{I}\left(\alpha_{i}^{r}-\beta_{i}^{r}\right) \frac{h_{i}}{a_{i, \infty}}\right)^{2} & \\
& =-\sum_{r=1}^{R} k_{f}^{r} a_{\infty}^{\alpha^{r}}\left(\left\langle\alpha^{r}-\beta^{r}, h\right\rangle\right)^{2} \leq 0
\end{aligned}
$$

we see that all eigenvalues of $L$ are contained in $(-\infty, 0]$. Finally, if there is $h \in \operatorname{Im} W^{\top}$ such that $L h=0$, the latter identity shows that

$$
\left\langle\alpha^{r}-\beta^{r}, h\right\rangle \quad \text { for all } r=1, \ldots, R \text {. }
$$


This shows that the vector $h=\left(h_{i}\right)_{i=1, \ldots, I}$ is perpendicular to the image of $W^{\top}$ in $\langle\cdot, \cdot\rangle$. Since $h$ is also in the image of $W^{\top}$, we deduce that $h=0$. Hence 0 is not an eigenvalue of $L$ in $\operatorname{Im} W^{\top}$, and all eigenvalues of $L$ in $\operatorname{Im} W^{\top}$ must be strictly negative. Since $L$ has a finite number of eigenvalues, the statement is proved (with no constructive estimate on $\lambda$ ).

Remark 6.3. Hypothesis 6.2 is not needed in the above result: the linearised operator always has a spectral gap once we avoid all conservations of the system. Hypothesis 6.2 is important later in order to show that the linearisation dominates the behaviour of the nonlinear equation.

Once we have the previous result, all remaining points of the proof of Theorem 2.1 for the four-species system are completely analogous in this general setting. We can write an analogue of Lemma 3.3, with a completely analogous proof which we omit:

Lemma 6.4. Assume the hypotheses of Theorem 6.1 and define the linear operator $T$ by (53), (55), (56). Define the norm $\|\cdot\|$ by (58) and denote by $\langle\cdot, \cdot\rangle$ the associated inner product. There exists $\lambda>0$ such that

$$
\langle h, T h\rangle \leq-\lambda\|h\|_{2}^{2}-\lambda\|\nabla h\|_{2}^{2}
$$

for all $h \in\left(L^{2}(\Omega)\right)^{I}$ satisfying

$$
\int_{\Omega} \mathbb{Q} h(x) \mathrm{d} x=0
$$

(equivalently, such that $\int_{\Omega} h \in \operatorname{Im} W^{\top}$ ). The constant $\lambda$ depends only on the dimension $d$, the domain $\Omega$, the numbers $a_{i, \infty}, i=1, \ldots, I$ and the diffusion constants $d_{i}, i=$ $1, \ldots, I$.

Remark 6.5. Again, Hypothesis 6.2 plays no role in the above result.

\subsection{Local $L^{p}$ stability}

The local $L^{2}$ stability of the nonlinear system (47)-(48) can the be obtained using the same proof as in Section 4. If $a=\left(a_{1}, \ldots, a_{I}\right)$ is a solution to (47)-(48) under the assumptions of Theorem 6.1 , calling $h=\left(h_{1}, \ldots, h_{I}\right)$ with

$$
h_{i}:=a_{i}-a_{i, \infty}, \quad i=1, \ldots, I,
$$

we can always write (47)-(48) as

$$
\partial_{t} h_{i}=d_{i} \Delta h_{i}+L_{i} h+N_{i} h, \quad i=1, \ldots, I,
$$

where $N_{i} h$ contains only quadratic monomials in $h_{j}, j=1, \ldots, I$ due to Hypothesis 6.2 . All arguments in Sections 4 and 5 can be reproduced in this case, obtaining Theorem 6.1 .

\section{Acknowledgements}

The authors are supported by the Spanish Ministerio de Economía y Competitividad and the European Regional Development Fund (ERDF/FEDER), project MTM201452056-P. We would like to thank Laurent Desvillettes, Klemens Fellner and Bảo Tăng for very helpful conversations on the paper. 


\section{References}

Amann, H. Global existence for semilinear parabolic systems. J. reine angew. Math, 360:47-83, 1985.

Brezis, H. Functional Analysis, Sobolev Spaces and Partial Differential Equations. Springer New York, New York, NY, 2010. ISBN 978-0-387-70913-0.

Cañizo, J. A., Desvillettes, L., and Fellner, K. Improved Duality Estimates and Applications to Reaction-Diffusion Equations. Communications in Partial Differential Equations, 39(6):1185-1204, May 2014. ISSN 1532-4133, arXiv:1304.4040.

Caputo, M. C. and Vasseur, A. Global regularity of solutions to systems of Reaction-Diffusion with Sub-Quadratic growth in any dimension. Communications in Partial Differential Equations, 34(10):1228-1250, September 2009.

Desvillettes, L. and Fellner, K. Exponential decay toward equilibrium via entropy methods for reaction-diffusion equations. Journal of Mathematical Analysis and Applications, 319(1):157-176, July 2006. ISSN 0022247X.

Desvillettes, L. and Fellner, K. Entropy methods for reaction-diffusion equations with degenerate diffusion arising in reversible chemistry. In Proceedings of EQUADIFF, 2007. Available at http://www.uni-graz.at/ fellnerk/preprints/ProcEquadiff.pdf [accessed October 2016].

Desvillettes, L. and Fellner, K. Entropy methods for reaction-diffusion equations: slowly growing a-priori bounds. Revista Matemática Iberoamericana, 24(2):407-431, 2008.

Desvillettes, L., Fellner, K., Pierre, M., and Vovelle, J. About global existence for quadratic systems of Reaction-Diffusion. Journal of Advanced Nonlinear Studies, 7 (3):491-511, 2007.

Desvillettes, L., Fellner, K., and Tang, B. Q. Trend to equilibrium for reaction-diffusion systems arising from complex balanced chemical reaction networks. SIAM Journal on Mathematical Analysis, 2017, arXiv:1604.04536.

Elias, J. Trend to equilibrium for a reaction-diffusion system modelling reversible enzyme reaction, October 2016, arXiv: 1610.07172.

Fellner, K. and Laamri, E.-H. Exponential decay towards equilibrium and global classical solutions for nonlinear reaction-diffusion systems. Journal of Evolution Equations, 16(3):681-704, January 2016. ISSN 1424-3202.

Fellner, K. and Tang, B. Q. Explicit exponential convergence to equilibrium for nonlinear reaction-diffusion systems with detailed balance condition. In Chen, L., Jüngel, A., and Desvillettes, L., editors, Special issue: Advances in reaction-cross-diffusion systems. Elsevier, February 2017, arXiv:1601.05992.

Fellner, K., Prager, W., and Tang, B. Q. The entropy method for reaction-diffusion systems without detailed balance: first order chemical reaction networks, April 2015, arXiv: 1504.08221. 
Fellner, K., Latos, E., and Suzuki, T. Global classical solutions for mass-conserving, (super)-quadratic reaction-diffusion systems in three and higher space dimensions. Discrete and Continuous Dynamical Systems - Series B, 21(10):3441-3462, December 2016. ISSN 1531-3492, arXiv:1511.04349.

Fischer, J. Global existence of renormalized solutions to entropy-dissipating reaction-diffusion systems. Archive for Rational Mechanics and Analysis, 218(1):553-587, 2015.

Fougères, P., Gentil, I., and Zegarlinski, B. Solution of a class of reaction-diffusion systems via logarithmic Sobolev inequality, May 2014, arXiv:1405.1170.

Friedman, A. Partial differential equations. Dover Publications, 2008. ISBN 9780486469195.

Gentil, I. and Zegarlinski, B. Asymptotic behaviour of reversible chemical reaction-diffusion equations. Kinetic and Related Models, 3(3):427-444, July 2010. ISSN 19375093.

Goudon, T. and Vasseur, A. Regularity analysis for systems of reaction-diffusion equations. Annales scientifiques de l'École Normale Supérieure, 43(1):117-142, 2010.

Horn, F. and Jackson, R. General mass action kinetics. Archive for Rational Mechanics and Analysis, 47(2):81-116, January 1972. ISSN 0003-9527.

Nirenberg, L. On elliptic partial differential equations. Annali della Scuola Normale Superiore di Pisa-Classe di Scienze, 13(2):115-162, 1959.

Rionero, S. A rigorous reduction of the $L^{2}$-stability of the solutions to a nonlinear binary reaction-diffusion system of PDE's to the stability of the solutions to a linear binary system of ODE's. Journal of Mathematical Analysis and Applications, 319(2): 377-397, July 2006. ISSN 0022-247X.

Rionero, S. $L^{2}$-energy decay of convective nonlinear PDEs reaction-diffusion systems via auxiliary ODEs systems. Ricerche di Matematica, 64(2):251-287, 2015.

Smoller, J. Shock waves and reaction-diffusion equations. Springer-Verlag, 1994. ISBN 9780387942599 .

María J. Cáceres, Departamento de Matemática Aplicada, Universidad de Granada, 18071 Granada, Spain. E-mail address: caceresg@ugr.es

José A. Cañizo, Departamento de Matemática Aplicada, Universidad de Granada, 18071

Granada, Spain. E-mail address: canizo@ugr.es 